\title{
Evaluating the use of fluorescence-based flow cytometry assay for dengue diagnosis using peripheral blood mononuclear cells
}

\author{
Luzia Aparecida Costa Barreira ${ }^{[1]}$, Priscila Santos Scheucher ${ }^{[2]}$, Marilia Farignoli Romeiro ${ }^{[1]}$, \\ Leonardo La Serra ${ }^{[1]}$, Soraya Jabur Badra ${ }^{[1]}$, William Marciel de Souza ${ }^{[1]}$ \\ and Luiz Tadeu Moraes Figueiredo ${ }^{[1]}$
}

[1]. Centro de Pesquisa em Virologia, Faculdade de Medicina de Ribeirão Preto, Universidade de São Paulo, Ribeirão Preto, SP, Brasil.

[2]. Laboratório de Hematologia, Faculdade de Medicina de Ribeirão Preto, Universidade de São Paulo, Ribeirão Preto, SP, Brasil.

\begin{abstract}
Introduction: Dengue virus (DENV) is the most important arthropod-borne viral disease worldwide with an estimated 50 million infections occurring each year. Methods: In this study, we present a flow cytometry assay (FACS) for diagnosing DENV, and compare its results with those of the non-structural protein 1 (NS1) immunochromatographic assay and reverse transcriptase polymerase chain reaction (RT-PCR). Results: All three assays identified 29.1\% (39/134) of the patients as denguepositive. The FACS approach and real-time RT-PCR detected the DENV in 39 and 44 samples, respectively. On the other hand, the immunochromatographic assay detected the NS1 protein in 40.1\% (56/134) of the patients. The Cohen's kappa coefficient analysis revealed a substantial agreement among the three methods. Conclusions: The FACS approach may be a useful alternative for dengue diagnosis and can be implemented in public and private laboratories.
\end{abstract}

Keywords: Dengue virus. Arbovirus. Flavivirus. Viral diagnostic. Flow cytometry.

\section{INTRODUCTION}

Dengue viruses (DENVs) are classified under the Flavivirus genus and the Flaviviridae family ${ }^{1}$. These viruses are enveloped positive-sense single-stranded RNA viruses that encode three structural proteins and seven non-structural proteins ${ }^{2}$. Based on the neutralization assay, DENVs are classified into four serotypes (DENV-1 to 4), and are transmitted to humans through the bite of infected Aedes mosquitoes ${ }^{1,3}$. Currently, DENV is the most important arthropod-borne viral disease worldwide, with an estimated 50 million infections occurring each year, which may be asymptomatic as in the majority of cases or may result in severe hemorrhagic fevers ${ }^{4}$. In Brazil, all four DENV serotypes have been reported in highly populated areas in the last three decades, such as DENV-1 in 1985, DENV-2 in 1990, DENV-3 in 2000, and DENV-4 in 2008, ${ }^{5,6}$. These viruses have spread and have been reintroduced probably several times, subsequently producing large outbreaks that affect at least ten million people, causing severe disease in tens of thousands of people, eventually leading to thousands of deaths ${ }^{5}$.

Corresponding author: $\mathrm{Dr}^{\mathrm{a}}$ Luzia Aparecida Costa Barreira.

e-mail: luziabarreira@gmail.com

Received 16 October 2017

Accepted 20 March 2018
Dengue infection usually begins as a mild acute febrile illness, but in some cases, it can progress to severe shock syndrome in a few hours ${ }^{4}$. Therefore, the diagnosis of dengue in the acute viremic phase of the disease is essential for suitable management of patients, especially at severe stages, and for consequent prognostic improvement ${ }^{1}$. Patients with dengue infection usually do not present specific antibodies during the acute phase of the disease. The diagnosis is based on isolation of the virus, detection of viral proteins (e.g., NS1 protein) in blood samples by immunochromatographic assay, and detection of the viral genome by RT-PCR ${ }^{7}$. Hence, to provide alternative methods of detection of DENV during the acute phase and for the subsequent effective management of patients, the development of quicker and easier diagnostic protocols for dengue is important. In this study, we have reported an assay for the diagnosis of DENVs in peripheral blood mononuclear cells (PBMCs) using a flow cytometry system with fluorescenceactivated cell sorting (FACS).

\section{METHODS}

\section{Study design and population}

During June to October 2015, 152 patients with acute febrile illness at Basic Health Units from the Ribeirão Preto City and the Jardinópolis municipality, São Paulo, Brazil, were recruited for the study. Samples were collected from the patients from 
the first to fifth day after the onset of symptoms. All participants had agreed and signed the consent form for this study.

\section{Ethical considerations}

This study was approved by the Research Ethics Committee of the Hospital of the School of Medicine of Ribeirão Preto, University of São Paulo (Process No. UPC 7641).

\section{Sample collection and preparation of PBMCs}

Blood samples were collected by venipuncture in heparin tubes BD Vacutainer ${ }^{\circledR} \mathrm{CPT}^{\mathrm{TM}}$ containing Ficoll-Hypaque solution (Becton Dickinson, USA). PBMCs were separated from whole blood based on Ficoll-Hypaque density gradient according to the manufacturer's instructions. PBMCs were carefully collected from the thin interface layer between red blood cells and plasma. The cells were then washed to remove platelets, and the PBMCs were suspended in $1 \mathrm{ml}$ of phosphatebuffered saline (PBS) solution and counted using the ABX Pentra 60 equipment (Horiba, Japan).

\section{FACS detection of DENVs from PBMCs}

To standardize the FACS approach for the detection of DENVs, human PBMCs from a dengue seronegative adult donor were suspended in $1 \mathrm{ml}$ of Roswell Park Memorial Institute (RPMI) 1640 medium (Sigma-Aldrich, USA) transferred into a 6-well tissue culture plate, and 3ml of RPMI 1640 medium (Sigma-Aldrich, USA) supplemented with 3\% fetal bovine serum and antibiotics (penicillin 100U/ml and streptomycin $100 \mathrm{mcg} / \mathrm{ml}$ ) was added per well. Subsequently, a total of $10^{6}$ PBMCs were infected with $3 \times 10^{4} \mathrm{PFU} / \mathrm{ml}$ of the DENV-4 strain $\mathrm{BeH} 402276$ and incubated at $37^{\circ} \mathrm{C}$ in an atmosphere of $5 \% \mathrm{CO}_{2}$ for six days. The DENV-4 strain BeH402276 was isolated from a patient with dengue fever on the $1^{\text {st }}$ of June, 1982 at Belém City by the Evandro Chagas Institute, Brazil and was kindly provided by Dr. Robert E. Shope from the University of Texas Medical Branch at Galveston. Subsequently, $100 \mu 1$ of PBMCs from healthy individuals was infected in vitro with DENVs, and the PBMCs from patients were incubated with $7 \mu 1$ of a mixture of murine ascitic fluids from animals hyper immunized with all four serotypes of DENV ${ }^{8}$. Then, PBMCs infected in vitro with DENVs and the PBMCs from patients were permeabilized and fixed with a FIX \& PERM Cell Permeabilization Kit (Invitrogen, USA) according to the manufacturer's instructions. Subsequently, the PBMCs were incubated for 20 minutes with $7 \mu \mathrm{l}$ of anti-mouse immunoglobulin $\mathrm{G}(\mathrm{IgG})$ antibodies conjugated with fluorescein isothiocyanate (FITC) (Sigma, USA) and submitted to the BD FACSCalibur ${ }^{\mathrm{TM}}$ platform (Becton Dickinson, USA).

The subpopulations of PBMCs infected in vitro with DENVs and the PBMCs from patients were fractionated by FACS using the following conjugated monoclonal antibodies capable of specifically recognizing different populations of white blood cells (WBCs): allophycocyanin-conjugated anti-CD3 antibodies bind to $\mathrm{T}$ cells, phycoerythrin-conjugated anti-CD4 antibodies to $\mathrm{T}$ helper cells, protein chlorophyllated piperidine-conjugated anti-CD8 antibodies to cytotoxic T cells, allophycocyaninconjugated anti-CD14 antibodies to monocytes, protein chlorophyllated piperidine-conjugated anti-CD19 antibodies to B lymphocytes, and phycoerythrin-conjugated anti-CD56 antibodies to natural killer cells (NKs)

The purity of the fractionated cells was evaluated by immunostaining for cell surface markers using a BD FACSCalibur ${ }^{\mathrm{TM}}$ platform (BD Biosciences, USA). The PBMCs infected in vitro with DENVs and the PBMCs from patients were analyzed after 8 hours of immunostaining in the BD FACSCalibur ${ }^{\mathrm{TM}}$ platform (BD Biosciences, USA) with a 488$\mathrm{nm}$ argon laser and Cell Quest TM Software (BD Biosciences, USA). Monocytes and lymphocytes were identified by their forward and side scatter characteristics, and enclosed in an electronic gate for fluorescence intensity analysis. Nonspecific fluorescence was reduced by using an antibody of the same isotype that was not specific to the antigenic sites of the samples in the analysis, and the background was also quantified in the negative control blood cells to avoid interference in the results. The results were calculated as the average percent of 10,000 single cell events in the analysis gate.

\section{Real-time RT-PCR, nested RT-PCR, and immunochromatographic assay}

The viral ribonucleic acid (RNA) in the blood samples was extracted with a QIAamp viral RNA extraction kit (Qiagen, Germany) and converted into double-stranded complementary deoxyribonucleic acid (cDNA) using Superscript III reverse transcriptase (Invitrogen, USA) according to the manufacturer's instructions. We then performed a real-time nested reverse transcription polymerase chain reaction (RT-PCR) generic to flaviviruses ${ }^{9}$, and a nested PCR to identify dengue serotypes ${ }^{10}$. In addition, all samples were tested for the presence of nonstructural protein 1 (NS1) using the immunochromatographic strip test (BioClin ${ }^{\mathrm{TM}}$, Brazil) for NS1 antigen capture according to the manufacturer's instructions. The inter-rater agreement for the three methods employed was measured by the Cohen's kappa coefficient ${ }^{11,12}$.

\section{RESULTS}

The FACS approach detected a total of 4.7\% DENV-positive cells from PBMCs infected in vitro with the DENV-4 strain BeH402276, and $0.7 \%$ DENV-positive cells in uninfected cells (negative control), which was considered as the background in this assay (Figure 1A and Figure 1B). These results were supported by real-time RT-PCR, which confirmed by amplification, the presence of the DENV viral genome in the PBMCs infected in vitro with DENV-4, but not in those of the uninfected sample.

The blood samples of 152 patients were tested by FACS and the NS1 immunochromatographic assay, including the 134 samples that were also examined by real-time RT-PCR. In the 134 samples tested by all assays, we observed a total of 29.1\% (39/134) DENV-positive patients (Figure 2A). The immunochromatographic assay showed the highest number of DENV-positive samples and detected the NS1 protein in $40.1 \%(56 / 134)$ of the patient samples (Figure 2A). The FACS approach and real-time RT-PCR presented positive confirmation for DENV in 39 and 44 samples, 


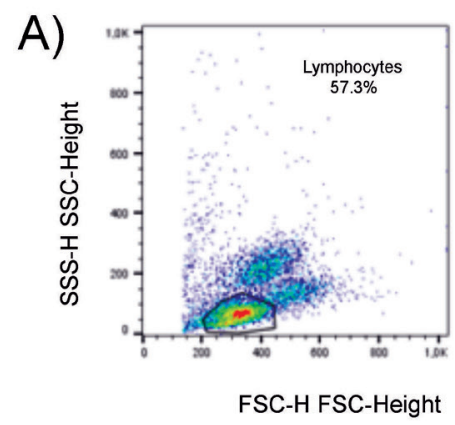

C)

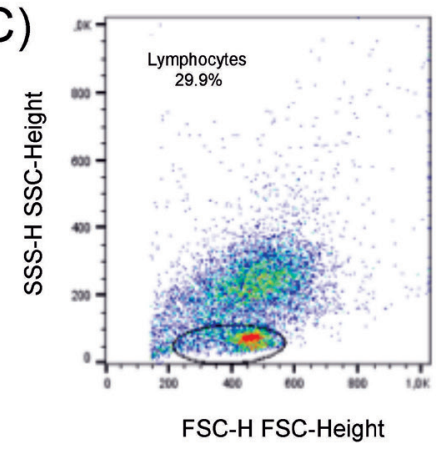

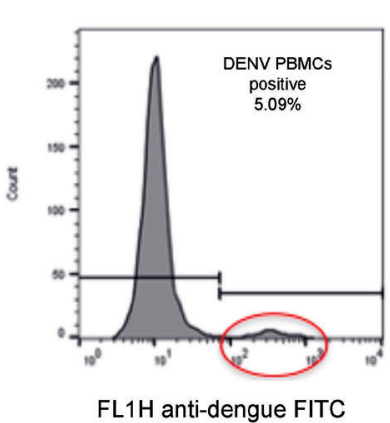

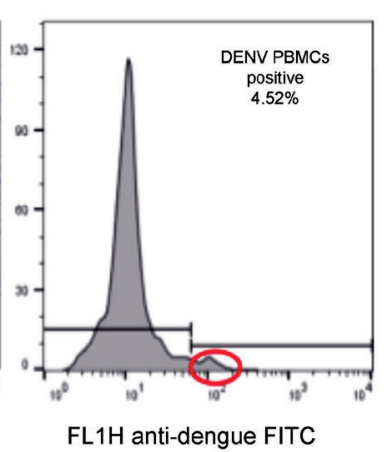

B)
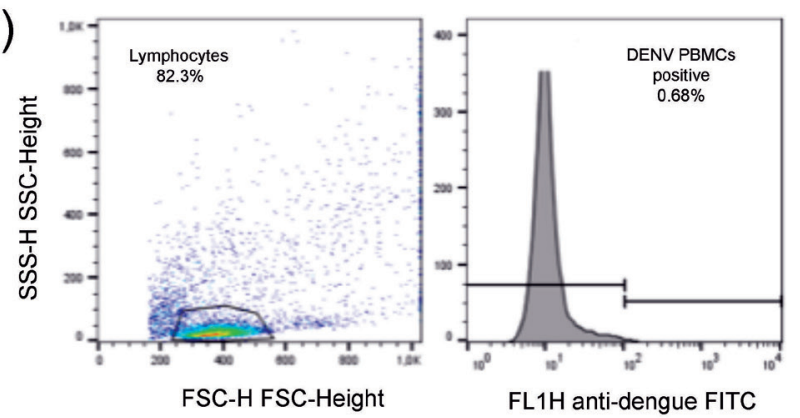

D)
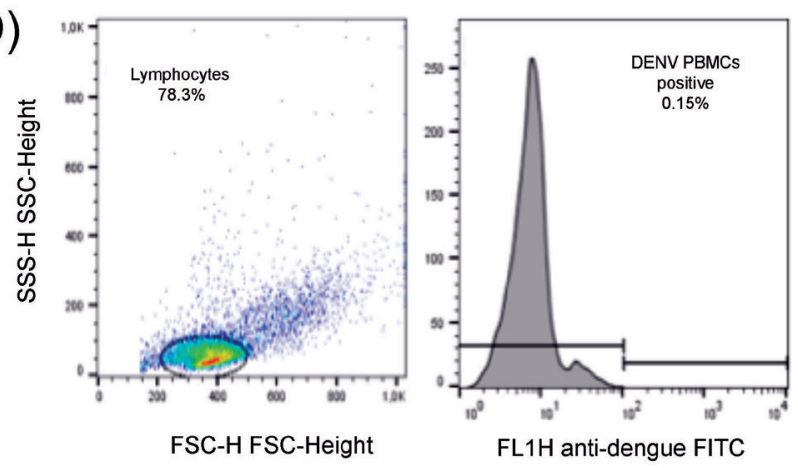

FIGURE 1: FACS-based diagnosis of dengue virus in PBMCs infected in vitro with dengue virus A) uninfected PBMCs, B) PBMCs of patients infected with dengue virus, C) and PBMCs of patients negative for dengue virus D). DENV: dengue virus; PBMCs: peripheral blood mononuclear cells; SSS-H: side scatter height; SSS-C: side scatter; FSC-H: forward scatter height; FL1H: intensity (height) in the flow cytometery channel 1; FITC: fluorescein isothiocyanate; FACS: flow cytometry assay.

respectively (Figure 2A). In addition, the Cohen's kappa coefficient showed a substantial agreement among the three methods (Figure 2A). Unfortunately, 18 samples could not be tested by real-time RT-PCR. Additionally, RT-nested PCR revealed that $95.5 \%(42 / 44)$ of the patients were infected with DENV-1 and 4.5\% (2/44) with DENV-2 (Figure 2B). All samples with a positive diagnosis of DENV showed more than $1.6 \%$ infected cells in the respective PBMCs by FACS (Figure 1C and Figure 1D). Interestingly, the $19 \mathrm{DENV}$-positive samples detected by the NS1 immunochromatographic assay had infected PBMCs, as identified by FACS, but showed less than $1.5 \%$ infected cells.

The patients infected with DENV-2 presented an average ratio of 3.6\% DENV infected PBMCs on the first day of febrile symptoms, but this ratio decreased to $2.0 \%$ in the samples collected on the fifth day after the initiation of clinical signs. The inverse correlation between the percentage of DENV infected PBMCs and the number of days after onset the disease was significant based on the Spearman correlation coefficient, which showed an $\mathrm{r}$ value of -0.96 ( $p$-value $=0.0043$ ). Interestingly, the subpopulations of PBMCs infected by DENV were predominantly $\mathrm{T}$ cells $(\mathrm{CD} 3+$ and $\mathrm{CD} 4+)$ at an average of $73.7 \%$, which also include the $32.1 \%$ of T helper cells. On the other hand, DENV positivity was detected at $8.5 \%$ in the B cells, $8.0 \%$ in the NK cells, and at $1.9 \%$ and $0.6 \%$ in the cytotoxic $\mathrm{T}$ cells and monocytes, which were the minority cell populations infected with DENVs, respectively.

\section{DISCUSSION}

Flow cytometry is used to detect and quantify viral antigens on the surface or within infected cells of human immunodeficiency virus (HIV), orthohantavirus, herpes simplex virus, rotavirus, and other viruses ${ }^{13-17}$. Additionally, this approach has been used to diagnose and titer flaviviruses of importance in veterinary and human public health, such as bovine viral diarrhea virus and DENV ${ }^{18-20}$. In this study, we showed that the DENV in clinical samples of patients with acute febrile symptoms can be rapidly detected using PBMCs by FACS, as demonstrated by the observed $29.1 \%$ DENV positivity that includes two patients with DENV-1 and 42 patients with DENV-2. This data demonstrates that FACS can detect all genotypes of DENV, in addition to DENV-4, which was used in the in vitro infection of PBMCs. This approach can be useful because PBMCs are the primary target cells in DENV infection ${ }^{21,22}$.

Our study showed that the subpopulations of PBMCs infected by DENV were predominantly $\mathrm{T}$ cells, especially $\mathrm{T}$ helper cells, and a representative proportion of $\mathrm{B}$ cells. This fact has also been reported in previous studies, which have demonstrated that $\mathrm{T}$ and $\mathrm{B}$ cells are the target cells in dengue infection $^{23-26}$. Another hypothesis suggests that monocytes are the primary targets in DENV infection and that $\mathrm{T}$ and $\mathrm{B}$ cells are not infected ${ }^{27,28}$. However, we did not observe this in our study, and in the contrary found that the smallest subpopulation 


\section{Cohen's kappa coecient $=0.725(0.879-0.571)$}

A p-value $(<0.001)$

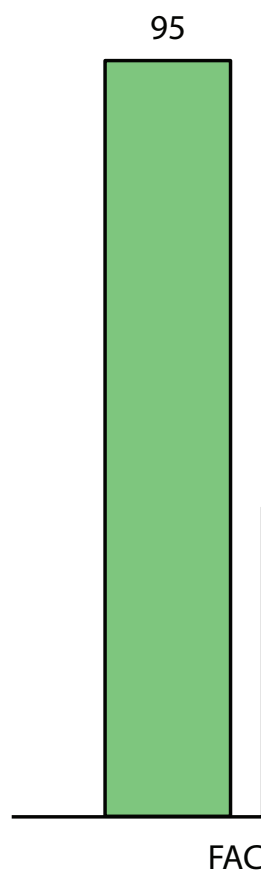

95

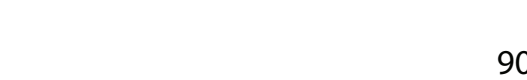

Positive

Negative

78

39

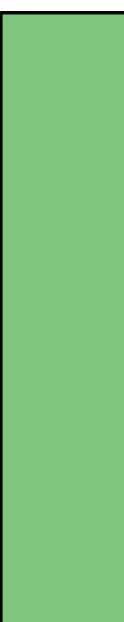

56

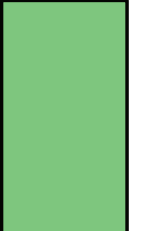

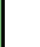

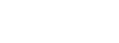

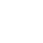

44

NS1

Real-time RT-PCR

B

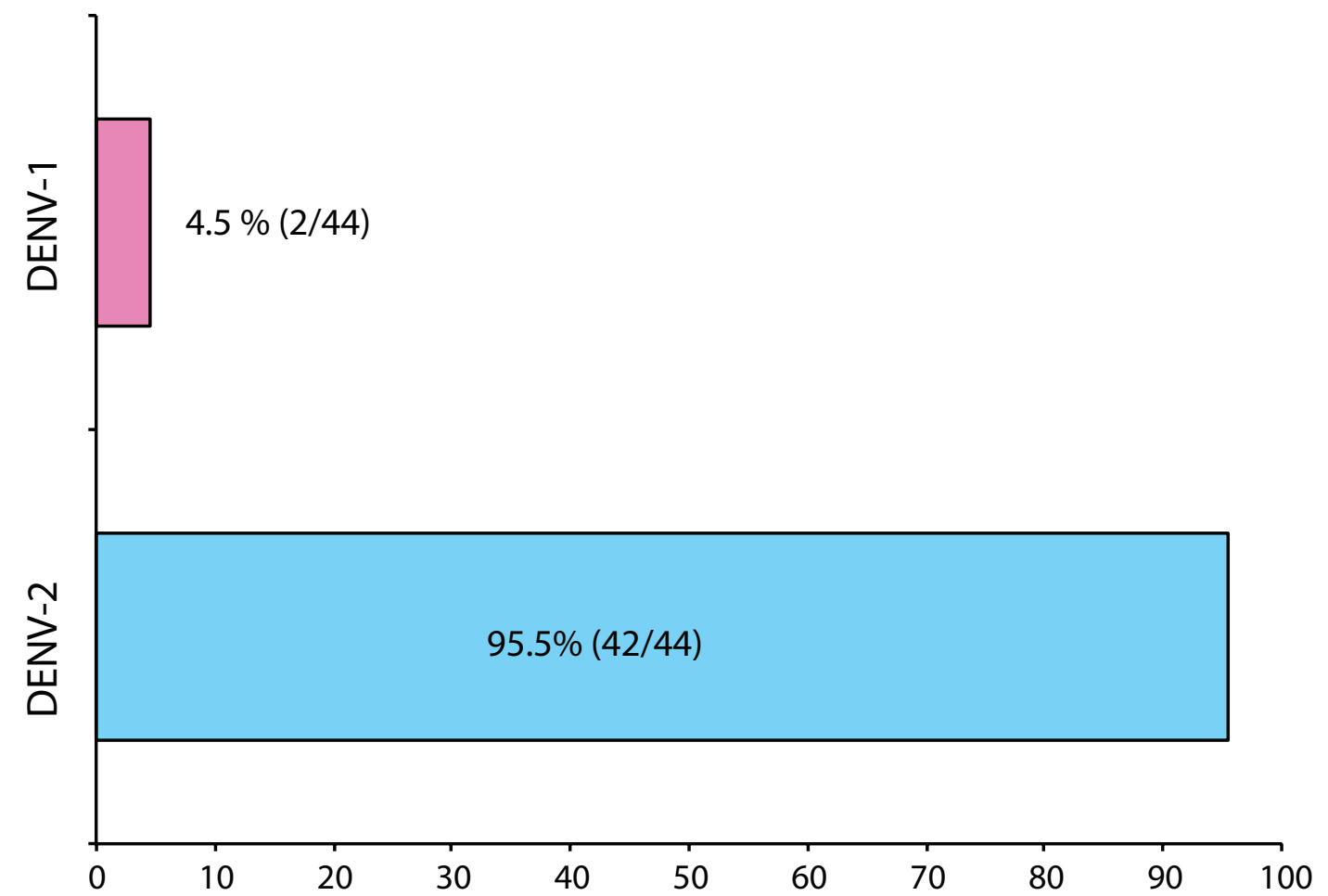

FIGURE 2: Frequency of dengue virus positivity in patients by FACS, NS1 and RT-PCR. A) Numbers of negative and dengue positive samples are highlighted in green and purple, respectively. B) The serotype of dengue-positive samples, dengue serotype-1 and 2, are highlighted in pink and blue, respectively. DENV: Dengue virus; FACS: flow cytometry assay; NS1: non-structural protein 1; RT-PCR: reverse transcriptase polymerase chain reaction. 
of PBMCs infected by DENV was monocytes, making up only $0.6 \%$ of the infected cells. Our results reinforce that T and B cells are the major target cells in dengue infection. However, the time and severity of infection, primary or secondary nature of infection, and genetic and immunity factors may affect the subpopulations cells of PBMCs ${ }^{25,26,29-31}$. In addition, further studies analyzing the mononuclear cells in primary and secondary dengue infections, as well as at varied severities of the infection may be important for elucidating the mechanism of dengue pathogenesis.

Results from all the diagnostic methods employed in this study showed a substantial agreement among them. The FACS approach to diagnose DENVs in PBMCs showed results similar to those obtained by RT-PCR with a concordance of $97.7 \%$. However, the FACS approach provides a quantification of the infected PBMCs that can be correlated to the DENV load in patients ${ }^{32}$. On the other hand, the FACS approach diagnosed a lower number of DENV-positive patients compared to the NS1 assay. This could be probably due to the multiple antigenic determinants that are expressed, both intracellularly and on the cell surface, in the NS1 protein, which is involved in enhancing viral RNA replication and also in the release of infectious viral particles ${ }^{19}$. Additionally, the temporal increase in antiviral immunity, including the appearance of neutralizing antibodies, causes reduced DENV viremia and probably decreases the number of infected cells in blood ${ }^{33}$.

In summary, the FACS approach using highly reactive antibodies that are specific to the antigenic components of DENVs may be quite useful for the diagnosis in outbreak, and surveillance of DENV in Brazil. This assay can also be implemented in public and private laboratories that have a large number of flow cytometers available for monitoring patients infected with HIV. Additionally, this assay enables quantification of the number of PBMCs infected by DENVs; thus, it can be easily implemented to monitor viral activity and as well as to measure viral load in pathogenesis studies.

\section{Conflict of interest}

The authors declare that there is no conflict of interest.

\section{Financial support}

This work was supported by the Fundação de Amparo à Pesquisa do Estado de São Paulo (FAPESP), Brazil (Grant number 14/02438-6 and Scholarship numbers 12/24150-9, 17/13981-0 and 14/20851-8).

\section{REFERENCES}

1. Martina BE, Koraka P, Osterhaus AD. Dengue virus pathogenesis: an integrated view. Clin Microbiol Rev. 2009;22(4):564-81.

2. Leitmeyer KC, Vaughn DW, Watts DM, Salas R, Villalobos I, de Chacon, et al. Dengue virus structural differences that correlate with pathogenesis. J Virol. 1999;73(6):4738-47.

3. Ramos-Castaneda J, Barreto dos Santos F, Martinez-Vega R, Galvão de Araujo JM, Joint G, Sarti E. Dengue in Latin America: systematic review of molecular epidemiological trends. PLoS Neg1 Trop Dis. 2017;11(1):e0005224.
4. Guzman MG, Halstead SB, Artsob H, Buchy P, Farrar J, Gubler DJ, et al. Dengue: a continuing global threat. Nat Rev Microbiol. 2010;8(suppl 12):S7-16.

5. Figueiredo LT. Serious disease outbreaks caused by viruses transmitted by Aedes aegypti in Brazil. Rev Soc Bras Med Trop. 2016;49(3):265-6.

6. Messina JP, Brady OJ, Scott TW, Zou C, Pigott DM, Duda KA, et al. Global spread of dengue virus types: mapping the 70 year history. Trends Microbiol. 2014;22(3):138-46.

7. Muller DA, Depelsenaire AC, Young PR. Clinical and laboratory diagnosis of dengue virus infection. J Infect Dis. 2017;215 (suppl 2):S89-S95.

8. Shope RE, Sather GE. Arboviruses. In: Lennette EH, Schimidt NJ, editors. Diagnostic procedures for viral, rickettsial and clamydial infections. $5^{\text {th }}$ edition. Washington: American Public Health Assn; 1979. p. $766-814$.

9. Romeiro MF, Souza WM, Tolardo AL, Vieira LC, Colombo TE, Aquino VH, et al. Evaluation and optimization of SYBR Green realtime reverse transcription polymerase chain reaction as a tool for diagnosis of the Flavivirus genus in Brazil. Rev Soc Bras Med Trop. 2016;49(3):279-85.

10. Lanciotti RS, Calisher CH, Gubler DJ, Chang GJ, Vorndam AV. Rapid detection and typing of dengue viruses from clinical samples by using reverse transcriptase-polymerase chain reaction. J Clin Microbiol. 1992;30(3):545-51.

11. Cohen J. A coefficient of agreement for nominal scales. Educ Psychol Meas. 1960;20(1):9.

12. Landis JR, Koch GG. The measurement of observer agreement for categorical data. Biometrics. 1977;33(1):159-74.

13. Barriga GP, Martinez-Valdebenito C, Galeno H, Ferrés M, Lozach PY, Tischler ND. A rapid method for infectivity titration of Andes hantavirus using flow cytometry. J Virol Methods. 2013;193(2):291-4.

14. Kung SH, Wang YC, Lin CH, Kuo RL, Liu WT. Rapid diagnosis and quantification of herpes simplex virus with a green fluorescent protein reporter system. J Virol Methods. 2000;90(2):205-12.

15. Brussaard CP, Marie D, Bratbak G. Flow cytometric detection of viruses. J Virol Methods. 2000;85(1-2):175-82.

16. Bosch A, Pinto RM, Comas J, Abad FX. Detection of infectious rotaviruses by flow cytometry. Methods Mol Biol. 2004;268:61-8.

17. McSharry JJ, Costantino R, Robbiano E, Echols R, Stevens R, Lehman JM. Detection and quantitation of human immunodeficiency virus-infected peripheral blood mononuclear cells by flow cytometry. J Clin Microbiol. 1990;28(4):724-33.

18. Qvist P, Aasted B, Bloch B, Meyling A, Rønsholt L, Houe H. Flow cytometric detection of bovine viral diarrhea virus in peripheral blood leukocytes of persistently infected cattle. Can J Vet Res. 1990;54(4):469-72.

19. Kao CL, Wu MC, Chiu YH, Lin JL, Wu YC, Yueh YY, et al. Flow cytometry compared with indirect immunofluorescence for rapid detection of dengue virus type 1 after amplification in tissue culture. J Clinical Microbiol. 2001;39(10):3672-7.

20. Lambeth CR, White LJ, Johnston RE, de Silva AM. Flow cytometrybased assay for titrating dengue virus. J Clinical Microbiol. 2005;43(7):3267-72.

21. Durbin AP, Vargas MJ, Wanionek K, Hammond SN, Gordon A, Rocha $\mathrm{C}$, et al. Phenotyping of peripheral blood mononuclear cells during acute dengue illness demonstrates infection and increased activation of monocytes in severe cases compared to classic dengue fever. Virology. 2008;376(2):429-5. 
22. Wang WK, Sung TL, Tsai YC, Kao CL, Chang SM, King CC. Detection of dengue virus replication in peripheral blood mononuclear cells from dengue virus type 2-infected patients by a reverse transcription-real-time PCR assay. J Clinical Microbiol. 2002;40(12):4472-8.

23. Lin YW, Wang KJ, Lei HY, Lin YS, Yeh TM, Liu HS, et al. Virus replication and cytokine production in dengue virus-infected human B lymphocytes. J Virol. 2002;76(23):12242-9.

24. Mongkolsapaya J, Dejnirattisai W, Xu XN, Vasanawathana S, Tangthawornchaikul N, Chairunsri A, et al. Original antigenic sin and apoptosis in the pathogenesis of dengue hemorrhagic fever. Nat Med. 2003;9(7):921-7.

25. Green S, Rothman A. Immunopathological mechanisms in dengue and dengue hemorrhagic fever. Curr Opin Infect Dis. 2006;19(5):429-36.

26. Rothman AL. Dengue: defining protective versus pathologic immunity. J Clinical Invest. 2004;113(7):946-51.

27. Blackley S, Kou Z, Chen H, Quinn M, Rose RC, Schlesinger JJ, et al. Primary human splenic macrophages, but not $\mathrm{T}$ or B cells, are the principal target cells for dengue virus infection in vitro. J Virol. 2007;81(24):13325-34.

28. Kou Z, Quinn M, Chen H, Rodrigo WW, Rose RC, Schlesinger JJ, et al. Monocytes, but not T or B cells, are the principal target cells for dengue virus (DV) infection among human peripheral blood mononuclear cells. J Medical Virol. 2008;80(1):134-46.

29. Baclig MO, Gervacio LT, Suarez LA, Buerano CC, Matias RR, Kumatori A, et al. Flow cytometric analysis of dengue virusinfected cells in peripheral blood. Southeast Asian J Trop Med Public Health. 2010;41(6):1352-8.

30. Thai KT, Wismeijer JA, Zumpolle C, de Jong MD, Kersten MJ, de Vries PJ. High incidence of peripheral blood plasmacytosis in patients with dengue virus infection. Clin Microbiology Infection. 2011;17(12):1823-8.

31. King AD, Nisalak A, Kalayanrooj S, Myint KS, Pattanapanyasat K, Nimmannitya S, et al. B cells are the principal circulating mononuclear cells infected by dengue virus. Southeast Asian J Trop Med Public Health. 1999;30(4):718-28.

32. Sydow FF, Santiago MA, Neves-Souza PC, Cerqueira DI, Gouvea AS, Lavatori MF, et al. Comparison of dengue infection in human mononuclear leukocytes with mosquito C6/36 and mammalian Vero cells using flow cytometry to detect virus antigen. Mem Inst Oswaldo Cruz. 2000;95(4):483-9.

33. Stephenson JR. Understanding dengue pathogenesis: implications for vaccine design. Bull World Health Organ. 2005;83(4):308-14. 